\title{
Inverse flux quantum periodicity of magnetoresistance oscillations in two-dimensional short-period surface superlattices
}

\author{
X. F. Wang, ${ }^{1}$ P. Vasilopoulos, ${ }^{1}$ and F. M. Peeters ${ }^{2}$ \\ ${ }^{1}$ Department of Physics, Concordia University, 1455 de Maisonneuve Ouest, Montréal, Québec, Canada H3G 1 M8 \\ ${ }^{2}$ Departement Natuurkunde, Universiteit Antwerpen (Campus Drie Eiken), B-2610 Antwerpen, Belgium
}

(Received 30 July 2003; revised manuscript received 15 October 2003; published 29 January 2004)

\begin{abstract}
Transport properties of the two-dimensional electron gas (2DEG) are considered in the presence of a perpendicular magnetic field $B$ and of a weak two-dimensional (2D) periodic potential modulation in the 2DEG plane. The symmetry of the latter is rectangular or hexagonal. The well-known solution of the corresponding tight-binding equation shows that each Landau level splits into several subbands when a rational number of flux quanta $h / e$ pierces the unit cell and that the corresponding gaps are exponentially small. Assuming the latter are closed due to disorder gives analytical wave functions and simplifies considerably the evaluation of the magnetoresistivity tensor $\rho_{\mu \nu}$. The relative phase of the oscillations in $\rho_{x x}$ and $\rho_{y y}$ depends on the modulation periods involved. For a $2 \mathrm{D}$ modulation with a short period $\leqslant 100 \mathrm{~nm}$, in addition to the Weiss oscillations the collisional contribution to the conductivity and consequently the tensor $\rho_{\mu \nu}$ show prominent peaks when one flux quantum h/e passes through an integral number of unit cells in good agreement with recent experiments. For periods $300-400 \mathrm{~nm}$ long used in early experiments, these peaks occur at fields 10 to 25 times smaller than those of the Weiss oscillations and are not resolved.
\end{abstract}

DOI: 10.1103/PhysRevB.69.035331

PACS number(s): 73.20.At, 73.21.-b, 73.61.-r

\section{INTRODUCTION}

In the last decade the magnetotransport of the twodimensional electron gas (2DEG), subjected to periodic potential modulations, has attracted considerable experimental ${ }^{1}$ and theoretical ${ }^{2,3}$ attention. For one-dimensional (1D) modulations novel oscillations of the magnetoresistivity tensor $\rho_{\mu \nu}$ have been observed, at low magnetic fields $B$, distinctly different in period and temperature dependence from the usual Shubnikov-de Haas ones observed at higher $B$. These novel oscillations reflect the commensurability between two length scales: the cyclotron diameter at the Fermi level $2 R_{c}$ $=2 \sqrt{2 \pi n_{e}} \ell^{2}$, where $n_{e}$ is the electron density, $\ell$ the magnetic length, and $a$ the period of the potential modulation. The situation is similar but less clear-cut for 2D modulations from both a theoretical ${ }^{4-7}$ and an experimental ${ }^{7-9}$ point of view. To date most of the experimental results pertinent to 2D modulations ${ }^{7-9}$ with square or hexagonal symmetry have indicated strongly that the predicted ${ }^{10}$ fine structure of the Landau levels is not resolved. Magnetotransport theories pertinent to this case are rather limited ${ }^{4,7,8}$ in contrast with those for 1D modulations.

Recent observations ${ }^{9}$ call for additional theoretical work since they could not be fully explained by earlier semiclassical theories. ${ }^{4}$ In this paper we develop, along the lines of Ref. 3, the relevant quantum-mechanical magnetotransport theory of the 2DEG for precisely the case that the fine structure of the Landau levels is not resolved. Our goal is to explain recent experimental results ${ }^{11}$ on $2 \mathrm{D}$, short-period ( $a$ $\sim 1000 \AA$ ) surface superlattices with mobility $\mu$ in the intermediate range, i.e., $\mu \sim 100 \mathrm{~m}^{2} / \mathrm{V}$ s. The symmetry of the $2 \mathrm{D}$ modulation is taken to be rectangular or hexagonal. A brief semiclassical account, pertinent to the former symmetry, was reported in Ref. 4(b). New magnetoresistance oscillations are found to occur when one flux quantum h/e passes through an integral number of unit cells as was recently observed experimentally. ${ }^{11}$ Here we show that these oscillations result from the interplay between band conduction and collisional conduction. A new contribution to the latter opens up as hopping between cyclotron orbits which are separated by an integral multiple of the modulation period and have the same position relative to the modulation lattice. This contribution is appreciable only in short-period superlattices and accordingly could not be resolved in early experiments on longperiod superlattices.

In the next section we derive the one-electron eigenfunctions and eigenvalues for rectangular and hexagonal modulations; we also present the density of states. The analytical and numerical results for the corresponding conductivity or resistivity components are presented in Sec. III. Numerical results are given in Sec. IV and concluding remarks in Sec. V.

\section{EIGENVECTORS, EIGENVALUES, AND DENSITY OF STATES}

We consider a 2DEG, in the $(x, y)$ plane, in the presence of a perpendicular magnetic field $\mathbf{B}=B \hat{z}$ and of a $2 \mathrm{D}$ periodic potential modulation $U(x, y)$. The electrons are considered as free particles with an effective mass $m^{*}$. In the absence of the modulation the normalized one-electron eigenfunction, in the gauge $\mathbf{A}=(0, B x, 0)$, is given by $e^{i k_{y} y} \phi_{n}\left(x+x_{0}\right) / \sqrt{L_{y}}$, where $\phi_{n}\left(x+x_{0}\right)$ is the well-known harmonic-oscillator function, centered at $-x_{0}=-\ell^{2} k_{y}$, and $L_{y}$ is the sample's width.

In the presence of a sinusoidal 1D modulation one can use perturbation theory ${ }^{2,3}$ to evaluate the energy spectrum and eigenfunctions. Alternatively, one can use a tight-binding scheme, along the lines of Ref. 10, and look for solutions of the one-electron Hamiltonian $H^{0}=(\mathbf{p}+e \mathbf{A})^{2} / 2 m^{*}$ 
$+V_{x} \cos \left(K_{x} x\right)$ that are linear combinations of the unperturbed $\left(V_{x}=0\right.$ ) ones: $\left|\varphi_{n k_{y}}\right\rangle=\Sigma_{p} A_{p}\left|n, k_{y}+p G\right\rangle$, where $G$ is a suitable wave vector introduced here for convenience and $\left|n, k_{y}+p G\right\rangle$ is the unperturbed state $\left(K_{x}=2 \pi / a_{x}\right.$, and $a_{x}$ is the modulation period along $x$ ). As in Ref. 10 we take $G$ $\equiv K_{y}=2 \pi / a_{y}$ with $a_{y}$ the modulation period along $y$. The summation over $p$ has to be extended to all integer $p$ values such that $-L_{x} / 2 \ell^{2} \leqslant k_{y}+p K_{y} \leqslant L_{x} / 2 \ell^{2}$, where $L_{x}$ is the length. For $p=0$ we have the limits for $k_{y}$ as $-a_{x} / 2 \ell^{2} \leqslant k_{y}$ $\leqslant a_{x} / 2 \ell^{2}$. Then the tight-binding equation $\left\langle n, k_{y}+p K_{y}\right| H^{0}$ $-E\left|\varphi_{n k_{y}}\right\rangle=0$, in which mixing of different Landau levels $n$ is neglected, gives acceptable solutions for the coefficients $A_{p}$ as $A_{p}=A_{0} \exp (i \xi p)$. The new states are labeled with the additional quantum number $\xi \quad(0 \leqslant \xi \leqslant 2 \pi):\left|\psi_{n k_{y} \xi}\right\rangle$ $=A_{0} \Sigma_{p} \exp (i \xi p)\left|n, k_{y}+p K_{y}\right\rangle$. The orthonormality condition $\left\langle\varphi_{n k_{y} \xi} \mid \varphi_{n k_{y} \xi^{\prime}}\right\rangle=\delta_{\xi \xi^{\prime}}$ gives $\xi=2 \pi \nu \ell^{2} K_{y} / L_{x} \rightarrow k_{x} \ell^{2} K_{y}, \quad \nu$ being an integer, and $A_{0}=\ell\left(K_{y} / L_{x}\right)^{1 / 2}$ by normalization. The energy spectrum obtained in this way is the same as that obtained by perturbation theory. ${ }^{2,3}$

We will now use this information to obtain the corresponding eigenvectors and eigenvalues for a 2D modulation potential.

\section{A. Rectangular symmetry}

We assume the following one-electron Hamiltonian:

$$
H^{0}=(\mathbf{p}+e \mathbf{A}) / 2 m^{*}+V_{x} \cos \left(K_{x} x\right)+V_{y} \cos \left(K_{y} y\right),
$$

where $K_{\mu}=2 \pi / a_{\mu}(\mu=x, y) ; a_{x}$ and $a_{y}$ are the periods along the $x$ and $y$ directions, respectively.

In the gauge chosen, $\mathbf{A}=(0, B x, 0)$, the second term of Eq. (1) is not diagonal in $k_{y}$ and therefore $\left|n, k_{y}\right\rangle$ is not a convenient basis set. But as in the 1D case we can look for solutions of Eq. (1) in the form $\left|\varphi_{n, k_{y}}\right\rangle=\Sigma_{p} A_{p}\left|n, k_{y}+p K_{y}\right\rangle$ as described above. This choice of eigenfunctions is also suggested by the fact that $V_{y} \cos K_{y} y$ connects the unperturbed state $\left|n k_{y}\right\rangle$ with only the states $\left|n, k_{y} \pm K_{y}\right\rangle$. In this case the equation $\left\langle n, k_{y}+p K_{y}\left|H^{0}-E\right| \varphi_{n, k_{y}}\right\rangle=0$, in which mixing of different Landau levels is neglected, takes the form

$$
\begin{aligned}
& V_{x} F_{n}\left(u_{x}\right) \cos \left(2 \pi p \alpha+K_{x} x_{0}\right) A_{p}+\frac{1}{2} V_{y} F_{n}\left(u_{y}\right)\left(A_{p+1}+A_{p-1}\right) \\
& \quad=\left(E-E_{n}\right) A_{p},
\end{aligned}
$$

where $\alpha=2 \pi \ell^{2} / a_{x} a_{y}, E_{n}=(n+1 / 2) \hbar \omega_{c}$ is the "unperturbed" eigenvalue, and $\omega_{c}=|e| B / m^{*}$ the cyclotron frequency. Further, $F_{n}\left(u_{\mu}\right)=\exp \left(-u_{\mu} / 2\right) L_{n}\left(u_{\mu}\right), L_{n}\left(u_{\mu}\right)$ is the Laguerre polynomial and $u_{\mu}=\ell^{2} K_{\mu}^{2} / 2$.

The solution of Eq. (2) gives the eigenvalues $E$ and the eigenvectors $A_{p}$. We see immediately that for $\alpha$ integer the equation admits the exponential solutions $A_{p}=A_{0} e^{i \xi p}$, with $A_{0}$ and $\xi$ given above. This is also the case for those values of $\alpha$ for which $F_{n}\left(u_{x}\right)$ vanishes since $u_{x}=2 \pi^{2} \ell^{2} / a_{x}^{2}$ $=\pi\left(a_{y} / a_{x}\right) \alpha$. In the former case we have

$$
E_{n k \xi}=E_{n}+V_{x} F_{n}\left(u_{x}\right) \cos \left(K_{x} x_{0}\right)+V_{y} F_{n}\left(u_{y}\right) \cos \xi
$$

and in the latter

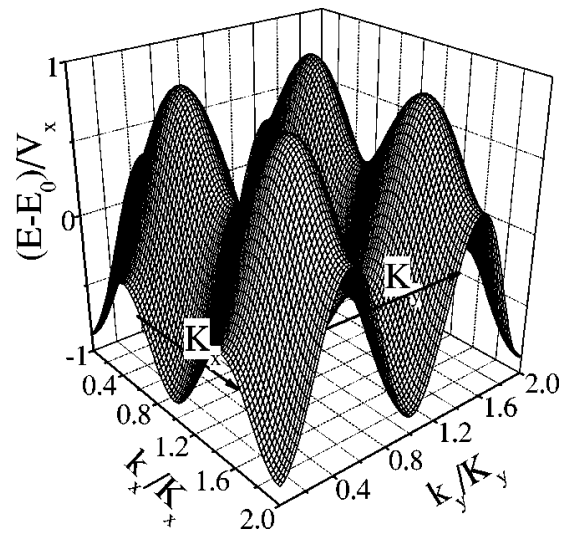

FIG. 1. Energy spectrum as a function of the wave vectors $k_{x}$ and $k_{y}$ for $n=0, \alpha=1, V_{x}=2 V_{y}=1 \mathrm{meV}$. The modulation wave vectors $K_{x}$ and $K_{y}$ are shown by the thick arrows.

$$
E_{n k \xi}=E_{n}+V_{y} F_{n}\left(u_{y}\right) \cos \xi,
$$

where $\xi=\left(2 \pi \nu / L_{x}\right) \ell^{2} K_{y} \equiv \ell^{2} K_{y} k_{x}$. In both cases the unperturbed Landau levels broaden into bands (with a bandwidth equal to $2\left[V_{x}\left|F_{n}\left(u_{x}\right)\right|+V_{y}\left|F_{n}\left(u_{y}\right)\right|\right]$ and $2 V_{y}\left|F_{n}\left(u_{y}\right)\right|$, respectively), which oscillate with magnetic field $B$ and (large) index $n$, cf. Refs. 2 and 3. The energy spectrum given by Eq. (3), plotted in Fig. 1 for $n=0, \alpha=1, V_{x}=2 V_{y}=1 \mathrm{meV}$, is a periodic function of $k_{x}$ and of $k_{y}$ since $\xi=\ell^{2} K_{y} k_{x}$ and $x_{0}$ $=\ell^{2} k_{y}$. Notice that the arguments of the cosines in Eq. (3) can be shifted by $2 \pi \alpha, \alpha$ integer.

One important consequence of this nonzero bandwidth is that the mean velocities $v_{x}$ and $v_{y}$, which vanish in the absence of modulation, are now finite: from $v_{\mu}$ $=d E_{n k \xi} / \hbar d k_{\mu}, \mu=x, y$, we obtain

$$
v_{x}=-\left(\ell^{2} K_{y} V_{y} / \hbar\right) F_{n}\left(u_{y}\right) \sin \xi
$$

and

$$
v_{y}=-\left(\ell^{2} K_{x} V_{x} / \hbar\right) F_{n}\left(u_{x}\right) \sin \left(K_{x} x_{0}\right) .
$$

Equations (5) and (6) lead to a finite diffusion or band conductivity which is absent when the modulation is not present, cf. Sec. II.

Equation (2) is the same as Harper's equation but the coefficients $V_{\mu} F_{n}\left(u_{\mu}\right)$ depend on the magnetic field. For $B$ values other than those pertaining to Eq. (3) it has been shown by Hofstadter ${ }^{12(a)}$ for the case of constant coefficients and by Claro and Wannier, ${ }^{12(b)}$ for the case that the latter depend on $B$ (hexagonal modulation), that the energy spectrum resulting from the numerical solution of Eq. (2), i.e., $E$, shows, when $E$ is measured in units of $V_{\mu} F_{n}\left(u_{\mu}\right)$, a nontrivial structure: for $\alpha=i / j, i, j$ being integers, each Landau level is split into $j$ subbands and Eq. (2) is periodic with period $j$. Here, in view of the reported experiments ${ }^{7-9}$ which did not indicate that this fine structure of the energy spectrum was resolved, we will assume that this is indeed the case, i.e., in samples of not exceptionally high mobilities, such as those of Ref. 5, the small gaps mentioned above are closed due to disorder and justify the assumption below, see Sec. II C. That is, we assume that the Landau levels are bands. Now from the numerical solution of Eq. (2) we know that the band- 
(a)
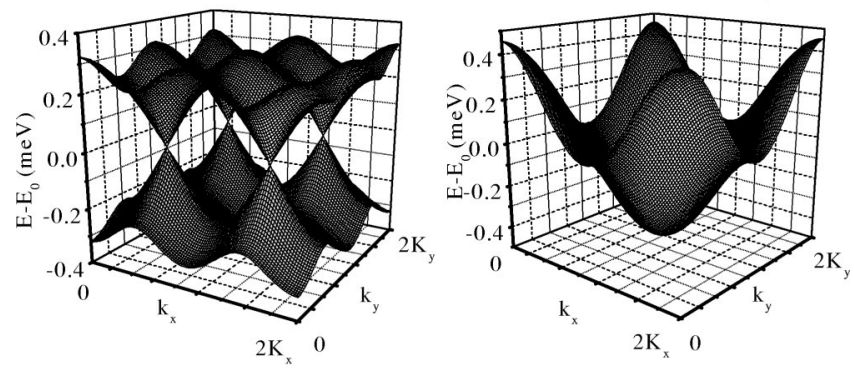

(c)
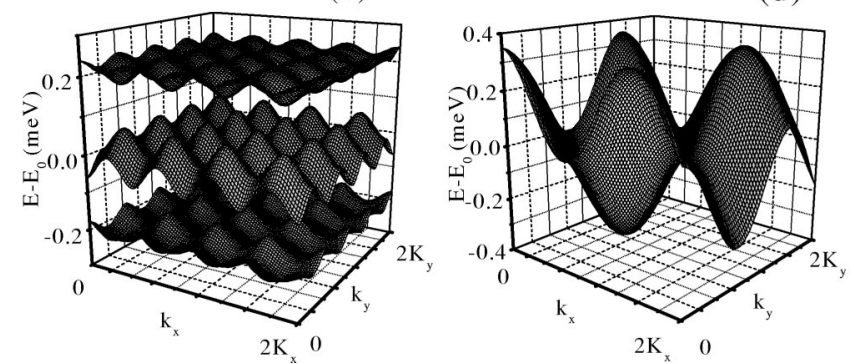

FIG. 2. Energy spectrum, obtained from the exact solution of Eq. (2), in (a) and (c), as a function of the wave vectors $k_{x}$ and $k_{y}$ for $n=0$. The corresponding approximate spectrum, given by Eq. (3), is shown in (b) and (d). In (a) and (b) we have $\alpha=1 / 2$ and $\alpha$ $=2 / 3$, in (c) and (d) $\alpha=2 / 3$. The periods $a_{x}=a_{y}=800 \AA$ pertain to the experiment of Ref. 11 .

width corresponding to $\alpha=i / j$ cannot exceed the value obtained from Eq. (3) or Eq. (4). Therefore, for computational convenience, we will assume that the energy spectrum is given by Eq. (3) and the eigenfunctions by $\left|\psi_{n k_{y} \xi}\right\rangle$ $=A_{0} \Sigma_{p} e^{i p \xi}\left|n, k_{y}+p K_{y}\right\rangle$.

In Fig. 2 we compare the energy spectrum obtained by exactly solving Eq. (2) with the one given by Eq. (3). We do so because in the conductivity calculations we will use Eq. (3), as an approximation that will be justified, for all magnetic fields or values of $\alpha$. The exact spectrum for $\alpha=i / j$ is composed of $j$ minibands. It's dependence on $k_{x}$, which does not appear in Eq. (2), is obtained by introducing appropriate new basis states, in the manner of Ref. 13, with $\left|k_{x}\right|$ $\leqslant \pi / i a_{x}$ and $\left|k_{y}\right| \leqslant \pi / a_{y}$ restricted in the magnetic Brillouin zone. As can be seen, the two spectra are quite different from each other. The corresponding difference in the density of states is much weaker if a small broadening is included, see Sec. II C below. For $\alpha$ integer, however, the exact spectrum and that given by Eq. (3) coincide; the result is shown in Fig. 1.

There are two alternative, approximate ways to obtain Eq. (3). First, we take $V_{y} \approx 0$ and use the corresponding 1D tightbinding states $\left|n, k_{y}, \xi\right\rangle$ to obtain the energy spectrum given by $E_{n}+V_{x} F_{n}\left(u_{x}\right) \cos \left(K_{x} x_{0}\right)$. We then use first-order perturbation theory, involving the states $\left|n k_{y} \xi\right\rangle$, to evaluate the energy correction to this spectrum due to the term $V_{y} \cos K_{y} y$ for $V_{y} \ll \hbar \omega_{c}+V_{x}$; the result is identical with that given by Eq. (3). Second, since these new oscillations of the magnetoresistance have been observed in weak magnetic fields and for weak modulations, we attempt a classical evaluation of the correction to the unperturbed energy $E_{n}$ by the modulation $V_{x} \cos K_{x} x+V_{y} \cos K_{y} y$ using the classical equations of motion $x(t)=x_{0}+R_{c} \sin \left(\omega_{c} t+\varphi\right), y(t)=y_{0}+R_{c} \cos \left(\omega_{c} t+\varphi\right) ; x_{0}$ and $y_{0}$ are the classical center coordinates, $R_{c}$ is the cyclotron radius, $\omega_{c}=|e| B / m^{*}$, and $\varphi$ is a phase factor. Without loss of generality we may take $\varphi=0$. Then, if $T$ is the period of the cyclotron motion, a straightforward evaluation gives

$$
\begin{aligned}
\langle U\rangle & =(1 / T) \int_{-T / 2}^{T / 2} d t\left[V_{x} \cos K_{x} x(t)+V_{y} \cos K_{y} y(t)\right] \\
& =V_{x} J_{0}\left(K_{x} R_{c}\right) \cos K_{x} x_{0}+V_{y} J_{0}\left(K_{y} R_{c}\right) \cos K_{y} y_{0},
\end{aligned}
$$

where $J_{0}(x)$ is the Bessel function of order zero. In the weak magnetic field limit $K_{\mu} R_{c} \gg 1$, Eq. (7) reduces to Eq. (3) for large $n$, i.e., for weak $B$. It is obvious that these three approximate ways of deriving the energy spectrum do not "see" its fine structure resulting from an exact numerical evaluation of the finite difference, Eq. (2), for $\alpha=i / j$. Therefore, they are applicable if the corresponding small gaps are closed due to disorder.

\section{B. Hexagonal symmetry}

We assume that the Hamiltonian is given by

$$
\begin{aligned}
H^{0}= & (\mathbf{p}+e \mathbf{A})^{2} / 2 m^{*}+V_{x} \cos K_{x} x \cos K_{y} y \\
& +V_{y}\left(1+\cos 2 K_{y} y\right) / 2 .
\end{aligned}
$$

For $V_{x}=V_{y}=V_{0}$ this reduces to the model studied experimentally by Fang and Stiles. ${ }^{7}$ If $x$ and $y$ are interchanged the energy spectrum, with $K_{y}=2 \pi / a$ and $K_{x}=2 \pi / \sqrt{3} a$, of the corresponding tight-binding equation has been studied numerically, for all values of the magnetic field, by Claro and Wannier ${ }^{12}$ and has the same structure as that of the square symmetry. Here, in line with the case of rectangular symmetry, we assume that the small gaps of the energy spectrum are closed due to disorder and use again the tight-binding description of Sec. II A. Corresponding to Eq. (2) we now obtain

$$
\begin{aligned}
& \frac{1}{2} V_{x} F_{n}\left(u_{x}+u_{y}\right)\left[\cos (2 \pi p \alpha+\gamma) A_{p+1}\right. \\
& \left.\quad+\cos (2 \pi p \alpha-\gamma) A_{p-1}\right]+\frac{V_{y}}{4} F_{n}\left(4 u_{y}\right)\left[A_{p+2}+A_{p-2}\right] \\
& \quad=\left(E-E_{n}-\frac{1}{2} V_{y}\right) A_{p}
\end{aligned}
$$

where $\gamma=K_{x} \ell^{2}\left(k_{y}+K_{y} / 2\right)$. When $\alpha$ is integer Eq. (9) has the solution $A_{p}=A_{0} \exp (i \xi p)$ with $A_{0}$ and $\xi$ given in Sec. II A and the eigenvalue $E$ is given by

$$
\begin{aligned}
E_{n k \xi}= & E_{n}+\frac{1}{2} V_{y}+V_{x} F_{n}\left(u_{x}+u_{y}\right) \cos \gamma \cos \xi \\
& +\frac{1}{2} V_{y} F_{n}\left(4 u_{y}\right) \cos 2 \xi
\end{aligned}
$$


where $\xi=\ell^{2} K_{y} k_{x}$. We notice that for $K_{x}=2 \pi / a$ and $K_{y}$ $=2 \pi \sqrt{3} a$, i.e., the usual hexagonal modulation, we have $F_{n}\left(u_{x}+u_{y}\right)=F_{n}\left(4 u_{y}\right)=F_{n}\left(8 \pi^{2} \ell^{2} / 3 a^{2}\right)$.

As in the rectangular case, we see that the Landau levels have broadened into bands with a bandwidth equal to $\left[2 V_{x}\left|F_{n}\left(u_{x}+u_{y}\right)+V_{y}\right| F_{n}\left(4 u_{y}\right) \mid\right]$ that oscillates with magnetic field and (large) $n$. Again the mean velocities $v_{x}$ and $v_{y}$ are finite

$$
\begin{aligned}
& v_{x}=-\left(V_{y} \ell^{2} K_{y} / \hbar\right) F_{n}\left(4 u_{y}\right) \sin 2 \xi-\left(V_{x} \ell^{2} K_{y} / \hbar\right) \\
& \times F_{n}\left(u_{x}+u_{y}\right) \cos \gamma \cos \xi \\
& v_{y}=-\left(V_{x} \ell^{2} K_{x} / \hbar\right) F_{n}\left(u_{x}+u_{y}\right) \sin \gamma \sin \xi ;
\end{aligned}
$$

this has important consequences for transport and will be detailed in the next section.

\section{The density of states}

The energy spectra given by Eqs. (3) and (10) are qualitatively different from the unmodulated spectrum, given by $E_{n}$, and from the corresponding 1D modulation spectrum, given by $E_{n}+F_{n}\left(u_{x}\right) \cos K_{x} x_{0}$. These differences are also reflected in the density of states (DOS) defined by $D(E)$ $=2 \Sigma_{n k_{y} \xi} \delta\left(E-E_{n k_{y} \xi}\right)$. For a 2D modulation with rectangular symmetry, corresponding to Eq. (3), the DOS becomes

$$
\begin{aligned}
D(E)= & D_{0} \sum_{n=0}^{\infty} \int_{0}^{2 \pi} d \xi\left\{\left[V_{x} F_{n}\left(u_{x}\right)\right]^{2}\right. \\
& \left.-\left[E-E_{n}-V_{y} F_{n}\left(u_{y}\right) \cos \xi\right]^{2}\right\}^{-1 / 2},
\end{aligned}
$$

while for the one with hexagonal symmetry, corresponding to Eq. (10), the DOS is given by

$$
\begin{aligned}
D(E)= & D_{0} \sum_{n=0}^{\infty} \int_{0}^{2 \pi} d \xi\left\{\left[V_{x} F_{n}\left(u_{x}+u_{y}\right) \cos \xi\right]^{2}\right. \\
& \left.-\left(E-E_{n}-\frac{V_{y}}{2}\left[1+F_{n}\left(4 u_{y}\right) \cos 2 \xi\right]\right)^{2}\right\}^{-1 / 2},
\end{aligned}
$$

where $D_{0}=L_{y} L_{x} / \pi^{3} \ell^{2}$. The quantities within the curly brackets in Eqs. (13) and (14) must be positive.

In Fig. 3 we plot the DOS, given by Eq. (13), for various values of the parameters $a_{x}, a_{y}, V_{x}$, and $V_{y}$. For comparison we also show the DOS (dash-dotted curve) corresponding to the 1D modulation. The latter exhibits Van Hove singularities at the edges of each Landau level (band) reflecting the 1D nature of the electron motion in this band, since $v_{x}$ $\neq 0$ while $v_{y}=0$, cf. Refs. 2 and 3 . This is not the case for the 2D modulation: the electron motion is two dimensional, since both $v_{x}$ and $v_{y}$ are different from zero, cf. Eqs. (5) and (6). That is, in the 2D case the DOS is finite, see also Refs. 4(b). As shown there, the DOS is qualitatively the same as the one shown in Fig. 3 if the periods are the same and the strengths are varied. This can be immediately deduced from the factors $V_{x} F_{n}\left(u_{x}\right)$ and $V_{y} F_{n}\left(u_{y}\right)$ that appear in Eqs. (3)

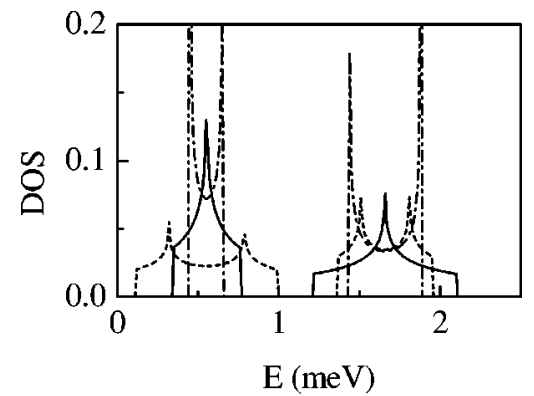

FIG. 3. Density of states vs energy for $V_{x}=V_{y}=0.5 \mathrm{meV}, a_{x}$ $=800 \AA$ with $a_{y}=800 \AA$ and $a_{y}=1600 \AA$ for the solid and dotted curves, respectively. The dash-dotted curve is the result for a $1 \mathrm{D}$ modulation along the $\mathrm{x}$ direction with the same period and modulation strength as in the $2 \mathrm{D}$ case. The magnetic field is $B$ $=0.64 \mathrm{~T}$.

and (11). The DOS for the hexagonal modulation is not shown since it is similar to the one shown in Fig. 3.

In Fig. 4 we compare the DOS obtained from the exact energy spectrum with that obtained using Eq. (3). In this comparison we include a level broadening by replacing $\delta(E)$ in the definition of $D(E)$ by $\pi \Gamma /\left(E^{2}+\Gamma^{2}\right)$. In Fig. 4(a) we show the influence of the level broadening on the DOS for $\alpha=2 / 3$ and different values of $\Gamma$ specified in the caption. As can be seen, the subband structure disappears with increasing $\Gamma$ and the exact and approximate result approach each other. That is, the gaps between the minibands in each Landau level are closed with increasing level broadening. Notice that this happens for quite small values of $\Gamma$ compared to the cyclotron energy which is about $1 \mathrm{meV}$ in this example. As shown in (c), the same behavior of the DOS occurs for $\alpha=1 / 2$. Notice also that this closeness between the exact and approximate DOS occurs despite the drastic difference in the

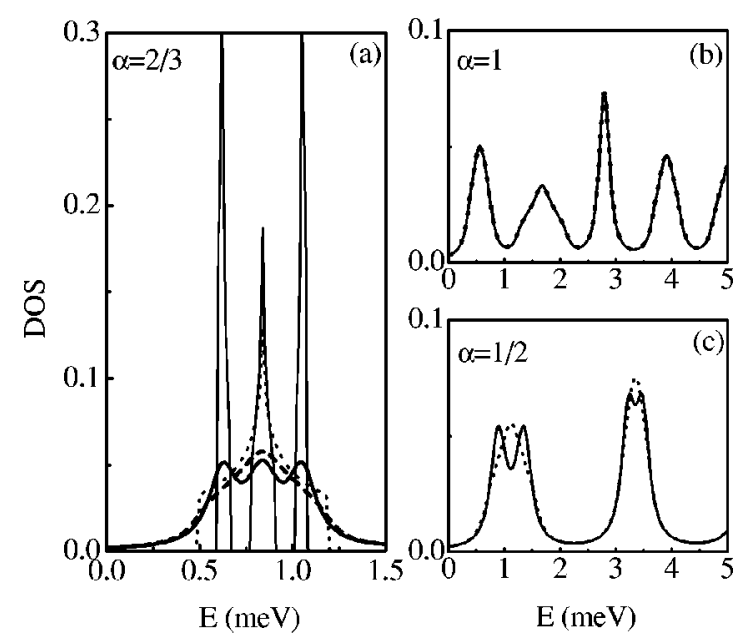

FIG. 4. (a) Density of states vs energy for $\alpha=2 / 3$ and energy level width $\Gamma=0$ (thin curves) and $\Gamma=1 \mathrm{~K}$ (thick curves). The solid and dotted curves are, respectively, the exact and approximate results. The DOS for $\alpha=1$ in (b) and $\alpha=1 / 2$ in (c) is plotted, respectively, for $\Gamma=1.1 \mathrm{~K}$ and $\Gamma=1.5 \mathrm{~K}$ obtained from $\Gamma$ $=\left(e \hbar / m^{*}\right) \sqrt{B / \pi \mu}$. The parameters used are the same as those in Fig. 2. 
corresponding energy spectra shown in Fig. 2. In addition, as shown in (b), for integer $\alpha$ the exact and approximate results for the DOS are identical.

\section{TRANSPORT COEFFICIENTS}

\section{A. Basic expressions}

For weak electric fields $E$, i.e., for linear responses, and weak scattering potentials the conductivity tensor $\sigma_{\mu \nu}(\omega)$ in the one-electron approximation has been evaluated in detail in Ref. 3: $\sigma_{\mu \nu}(\omega)=\sigma_{\mu \nu}^{d}(\omega)+\sigma_{\mu \nu}^{n d}(\omega), \mu, \nu=x, y$. The contribution $\sigma_{\mu \nu}^{d}(\omega)$ stems from the diagonal part of the density operator $\rho$. In a suitable basis $\left\langle J_{\mu}^{d}\right\rangle=\operatorname{Tr}\left(\rho^{d} J_{\mu}\right)=\sigma_{\mu \nu}^{d} E_{\nu}$, where $J_{\mu}$ is the current density and $\sigma_{\mu \nu}^{n d}(\omega)$ comes from the nondiagonal part of $\rho\left(\rho=\rho^{d}+\rho^{n d}\right)$. In general $\sigma_{\mu \nu}^{d}(\omega)$ $=\sigma_{\mu \nu}^{d i f}(\omega)+\sigma_{\mu \nu}^{c o l}(\omega)$, where $\sigma_{\mu \nu}^{\text {dif }}(\omega)$ indicates diffusive contributions and $\sigma_{\mu \nu}^{c o l}(\omega)$ collisional contributions. For the diffusive contribution we have

$$
\sigma_{\mu \nu}^{d i f}(0)=\frac{\beta e^{2}}{\Omega} \sum_{\zeta} f_{\zeta}\left(1-f_{\zeta}\right) \tau\left(E_{\zeta}\right) v_{\mu}^{\zeta} v_{\nu}^{\zeta},
$$

provided that the scattering is elastic or quasielastic, and for the collisional one

$$
\sigma_{\mu \nu}^{c o l}(0)=\frac{e^{2}}{2 \Omega} \sum_{\zeta \zeta^{\prime}} f_{\zeta}\left(1-f_{\zeta^{\prime}}\right) W_{\zeta \zeta^{\prime}}\left(\alpha_{\mu}^{\zeta}-\alpha_{\mu}^{\zeta^{\prime}}\right)^{2},
$$

for both elastic $\left(f_{\zeta}=f_{\zeta^{\prime}}\right)$ and inelastic $\left(f_{\zeta} \neq f_{\zeta^{\prime}}\right)$ scattering. $W_{\zeta \zeta^{\prime}}$ is the transition rate between the unperturbed oneelectron states $|\zeta\rangle$ and $\left|\zeta^{\prime}\right\rangle, \Omega$ the volume of the system, $e$ the electron charge, $\tau\left(E_{\zeta}\right)$ the relaxation time, and $\alpha_{\mu}^{\zeta}$ $=\left\langle\zeta\left|r_{\mu}\right| \zeta\right\rangle$ the mean value of the $\mu$ component of the position operator when the electron is in state $|\zeta\rangle$ and has velocity $v_{\mu}^{\zeta}=\left\langle\zeta\left|v_{\mu}\right| \zeta\right\rangle$. Equation (15) describes transport through extended states whereas Eq. (16) deals with transport through localized states and is absent in semiclassical treatments.

The nondiagonal contribution $\sigma_{\mu \nu}^{n d}(\omega)$ to the conductivity is given by

$$
\begin{aligned}
\sigma_{\mu \nu}^{n d}(\omega)= & \frac{2 i \hbar e^{2}}{\Omega} \sum_{\zeta \neq \zeta^{\prime}} f_{\zeta}\left(1-f_{\zeta^{\prime}}\right)\left\langle\zeta\left|v_{\mu}\right| \zeta^{\prime}\right\rangle \\
& \times\left\langle\zeta^{\prime}\left|v_{\nu}\right| \zeta\right\rangle \frac{1-e^{\beta\left(E_{\zeta^{\prime}}-E_{\zeta^{\prime}}\right)}}{E_{\zeta}-E_{\zeta^{\prime}}} \lim _{\epsilon \rightarrow 0} \frac{1}{E_{\zeta}-E_{\zeta^{\prime}}+\hbar \omega+i \epsilon} .
\end{aligned}
$$

If we use the identity $f_{\zeta}\left(1-f_{\zeta^{\prime}}\right) \exp \left[\beta\left(E_{\zeta}-E_{\zeta^{\prime}}\right)\right]=f_{\zeta^{\prime}}(1$ $-f_{\zeta}$ ), Eq. (17) takes the form of the well-known KuboGreenwood formula.

Apart from their use in Ref. 3 for the 1D modulation case, the above formulas have also been successfully applied to various situations of electronic transport, such as hopping conduction, ${ }^{14(\mathrm{a})}$ Aharonov-Bohm effect, ${ }^{14(\mathrm{~b})}$ quantum Hall effect, ${ }^{14(c)}$ etc.
The resistivity tensor $\rho_{\mu \nu}$ is given in terms of the conductivity tensor $\rho=\sigma^{-1}$. We will use the standard expressions $\rho_{x x}=\sigma_{y y} / S, \rho_{y y}=\sigma_{x x} / S$, and $\rho_{y x}=-\rho_{x y}=-\sigma_{y x} / S$ with $S$ $=\sigma_{x x} \sigma_{y y}-\sigma_{x y} \sigma_{y x}$.

\section{B. Analytical evaluations}

The scattering mechanism enters the conductivity expressions (15) and (16) through the relaxation time $\tau\left(E_{\zeta}\right)$ and the transition rate $W_{\zeta \zeta^{\prime}}$, respectively; in contrast, Eq. (17) is independent of the scattering when the latter is weak. ${ }^{14}$

We assume that the electrons are scattered elastically by randomly distributed impurities. This type of scattering is dominant at the low temperatures of the reported experiments. Further, we expand the impurity potential in Fourier components, i.e., $U(\mathbf{r}-\mathbf{R})=\sum \mathbf{q} U_{\mathbf{q}} \exp [i \mathbf{q} \cdot(\mathbf{r}-\mathbf{R})]$, with $U_{\mathbf{q}}=2 \pi e^{2} / \epsilon\left(q^{2}+k_{s}^{2}\right)^{1 / 2}$ corresponding to the screened impurity potential $U(\mathbf{r})=\left(e^{2} / \epsilon r\right) \exp \left(-k_{s} r\right) ; \mathbf{r}$ and $\mathbf{R}$ are the electron and impurity positions, respectively, $\mathbf{q}=q_{x} \hat{x}+q_{y} \hat{y}$, $\epsilon$ is the dielectric constant, and $k_{s}$ the screening wave vector.

Diffusive contribution. For weak modulation potentials $V_{x}$ and $V_{y}$, which is pertinent to most of the reported experiments, we may use $|\zeta\rangle=\left|n, k_{y}, \xi\right\rangle$ to evaluate the velocity matrix elements appearing in Eq. (15); the latter are given by Eqs. (5) and (6) for rectangular symmetry and by Eqs. (11) and (12) for hexagonal symmetry. As for the relaxation time $\tau\left(E_{\zeta}\right)$, it is defined by $1 / \tau\left(E_{\zeta}\right)=\Sigma_{\zeta^{\prime}} W_{\zeta \zeta^{\prime}}\left(v_{\zeta}-v_{\zeta}^{\prime}\right) / v_{\zeta}$. Though the Landau levels broaden into bands, this definition fails at the flat-band conditions, when $v_{\zeta}=v_{\zeta}^{\prime}=0$. For this reason we estimate it from the lifetime given by $1 / \tau\left(E_{\zeta}\right)$ $=\Sigma_{\zeta^{\prime}} W_{\zeta \zeta^{\prime}}$. In the limit $k_{s} \gg q$, we obtain $\tau=\tau\left(E_{\zeta}\right)$ $\approx\left(\pi \ell^{2} \hbar^{2} / N_{I} U_{0}^{2}\right)^{1 / 2}$, where $N_{I}$ is the $2 \mathrm{D}$ impurity density and $U_{0} \approx 2 \pi e^{2} / \epsilon k_{s}$. However, at weak magnetic fields we may use $\tau$ as constant and estimate it from the zero-field mobility $\mu: \tau=\tau_{0}=\mu m^{*} / e$.

We now use Eqs. (5), (6), and (15) with $\Sigma_{k}$ $\rightarrow\left(L_{y} / \pi\right) \int_{0}^{a_{x} / 2 \ell^{2}} d k_{y}$ and $\Sigma_{\xi} \rightarrow\left(L_{x} / \pi a_{x}\right) \int_{0}^{2 \pi} d \xi$. The result for $\sigma_{x x}^{d i f}$ is

$$
\begin{aligned}
\sigma_{x x}^{d i f} \approx & \frac{e^{2}}{h} \frac{\beta \tau}{\hbar \pi a_{x}} K_{y}^{2} \ell^{4} V_{y}^{2} \sum_{n} e^{-u_{y}\left[L_{n}\left(u_{y}\right)\right]^{2}} \\
& \times \int_{0}^{2 \pi} d \xi \int_{0}^{a_{x} /} \ell^{2} d k_{y} f_{n k_{y} \xi}\left(1-f_{n k_{y} \xi}\right) \sin ^{2} \xi .
\end{aligned}
$$

The component $\sigma_{y y}^{d i f}$ is given by Eq. (18) with $x$ and $y$ interchanged, and $\sin ^{2} \xi$ replaced by $\sin ^{2}\left(\ell^{2} K_{x} k_{y}\right)=\sin ^{2}\left(K_{x} x_{0}\right)$. In the limit of vanishing $V_{y}$ Eq. (18) gives the result of a $1 \mathrm{D}$ modulation, $\sigma_{x x}^{d i f}=0$. If we neglect the weak $k_{y}$ and $\xi$ dependence of the factor $f_{n k_{y} \xi}\left(1-f_{n k_{y} \xi}\right)$ we obtain the simplified expression

$$
\sigma_{x x}^{d i f} \approx \frac{e^{2}}{h} \frac{\beta \tau}{\hbar} K_{y}^{2} \ell^{2} V_{y}^{2} \sum_{n} e^{-u_{y}\left[L_{n}\left(u_{y}\right)\right]^{2} f_{n}\left(1-f_{n}\right) .}
$$

The corresponding expression for $\sigma_{y y}^{d i f}$ is given by Eq. (19) with $x$ and $y$ interchanged. 
For hexagonal symmetry we use $K_{x}=2 \pi / a$ and $K_{y}$ $=2 \pi / \sqrt{3} a$. The results for $\sigma_{x x}^{d i f}$ and $\sigma_{y y}^{d i f}$ are similar to Eq. (18) and can be easily obtained using Eqs. (11) and (12) for the velocities. The result for $\sigma_{y y}^{d i f}$, corresponding to Eq. (19), is given by Eq. (19) with $K_{y}^{2} V_{y}^{2}$ replaced by $K_{x}^{2} V_{x}^{2} / 2$ and that for $\sigma_{x x}^{d i f}$ by

$$
\sigma_{x x}^{d i f} \approx \pi \frac{e^{2}}{h} \frac{\beta \tau}{\hbar} K_{y}^{2} V_{y}^{2}\left(1+\frac{V_{x}^{2}}{2 V_{y}^{2}}\right) e^{-u} \sum_{n}\left[L_{n}(u)\right]^{2} f_{n}\left(1-f_{n}\right),
$$

where $u=8 \pi^{2} \ell^{2} / 3 a^{2}$.

Collisional contribution. To evaluate this contribution to order $V_{\mu}^{2}$ we must use the perturbed wave function to order $V_{\mu}$. The procedure for evaluating Eq. (16) is identical with that corresponding to the $1 \mathrm{D}$ modulation detailed previously. ${ }^{3}$ We have again $\langle\zeta|x| \zeta\rangle-\left\langle\zeta^{\prime}|x| \zeta^{\prime}\right\rangle=\ell^{2}\left(k_{y}\right.$ $\left.-k_{y}^{\prime}\right)$; the only new ingredient are the following matrix elements:

$$
\left\langle n k_{y} \xi|y| n k_{y} \xi\right\rangle=-\xi / K_{y}
$$

and

$$
\begin{aligned}
\left|\left\langle n k_{y} \xi\left|e^{i \mathbf{q} \cdot \mathbf{r}}\right| n^{\prime} k_{y}^{\prime} \xi^{\prime}\right\rangle\right|^{2}= & \left(n ! / n^{\prime} !\right) u^{n^{\prime}-n} e^{-u} \\
& \times\left[L_{n}^{n^{\prime}-n}(u)\right]^{2} \delta_{\xi, \xi^{\prime}+c_{y} q_{x}} \delta_{k_{y}, k_{y}^{\prime}-q_{y}},
\end{aligned}
$$

where $u=\ell^{2}\left(q_{x}^{2}+q_{y}^{2}\right) / 2$ and $c_{y}=\ell^{2} K_{y}$.

We now use Eqs. (16), (20), and (21), and the standard expression for the transition rate

$$
W_{\zeta \zeta^{\prime}}=\sum_{\mathbf{q}} U_{\mathbf{q}}^{2}\left|\left\langle n k_{y} \xi\left|e^{i \mathbf{q} \cdot \mathbf{r}}\right| n^{\prime} k_{y}^{\prime} \xi^{\prime}\right\rangle\right|^{2} \delta\left(E_{n k_{y} \xi}-E_{n^{\prime} k_{y}^{\prime} \xi^{\prime}}\right) .
$$

We use the spectrum (3) and shift the argument of the cosines by $\ell^{2} K_{x} K_{y}=2 \pi \alpha, \alpha$ integer, in the $\delta$ function as well as in the factor $f_{n k_{y} \xi}\left(1-f_{n^{\prime} k_{y}^{\prime} \xi^{\prime}}\right)$. Then Eq. (16) takes the form

$$
\begin{aligned}
\sigma_{y y}^{c o l} \approx & \frac{e^{2}}{h} \frac{\beta N_{I} U_{0}^{2}}{4 a_{x}} \sum_{n, n^{\prime}} \int_{0}^{\infty} d u e^{-u} u^{n^{\prime}-n+1}\left[L_{n}^{n^{\prime}-n}(u)\right]^{2} \\
& \times \int_{0}^{2 \pi} d \xi \int_{0}^{a_{x} l} \ell^{2} d k_{y} f_{n k_{y} \xi} \\
& \times\left(1-f_{n^{\prime}, k_{y}+K_{y}+q_{y}, \xi-c_{y}\left(K_{x}+q_{x}\right)}\right) \\
& \times \delta\left(E_{n k_{y} \xi}-E_{n^{\prime}, k_{y}+K_{y}+q_{y}, \xi-c_{y}\left(K_{x}+q_{x}\right)}\right) .
\end{aligned}
$$

We proceed as follows. For weak magnetic fields involved in the problem the Landau-level index $n$ is large and the major contributions to the sum over $n^{\prime}$ come from $n^{\prime}$ values close $n$. With the asymptotic expansion of the Laguerre polynomials, $e^{-u / 2} L_{n}(u) \approx\left(\pi^{2} n u\right)^{-1 / 4} \cos (2 \sqrt{n u}-\pi / 4)$, it is an excellent approximation to take $F_{n}\left(u_{\mu}\right) \approx F_{n^{\prime}}\left(u_{\mu}\right)$. Then the $\delta$ function becomes

$$
\begin{aligned}
& \delta\left(E_{n k_{y} \xi}-E_{n^{\prime}, k_{y}+K_{y}+q_{y}, \xi-c_{y}\left(K_{x}+q_{x}\right)}\right) \\
& \approx \delta\left(\left(n-n^{\prime}\right) \hbar \omega_{c}+2 F_{n}\left(u_{x}\right) V_{x} \sin c_{x}\left(K_{y}-q_{y} / 2\right)\right. \\
& \times \sin c_{x}\left(k_{y}+K_{y}-q_{y} / 2\right)+2 F_{n}\left(u_{y}\right) V_{y} \\
&\left.\times \sin c_{y}\left(K_{x}-q_{x} / 2\right) \sin c_{y}\left(k_{x}+K_{x}-q_{x} / 2\right)\right) .
\end{aligned}
$$

The shift by $\ell^{2} K_{x} K_{y}=2 \pi \alpha, \alpha$ integer, in Eq. (25) and in the factor $f_{n k_{y} \xi}\left(1-f_{n^{\prime} k_{v}^{\prime} \xi^{\prime}}\right)$ was made to stress the formal validity of Eqs. (24) and (25) for $\alpha$ integer. If we do not make it, we must put $K_{x}=K_{y}=0$ in the sine factors and change $E_{n^{\prime}, k_{y}+K_{y}+q_{y}, \xi^{-}-c_{y}\left(K_{x}+q_{x}\right)}$ to $E_{n^{\prime}, k_{y}+q_{y}, \xi-c_{y} q_{x}}$ wherever it appears. For $\alpha$ close to an integer though one can reinstate $K_{x}$ and $K_{y}$ in Eqs. (24) and (25) as shown.

We now remark that the largest contribution to the integral over $u$ in Eq. (24) comes from very small values of $q_{x}$ and $q_{y}$ due to the factor $\exp (-u)$ or the factor $1 / \sqrt{\pi^{2} n u}$ in the asymptotic expression $e^{-u}\left[L_{n}(u)\right]^{2} \approx \cos ^{2}(2 \sqrt{n u}$ $-\pi / 4) / \sqrt{\pi^{2} n u}$. In addition, for the usual 2D systems we have $k_{s} \approx 10^{8} / \mathrm{m}$ which is much larger than these small values of $q_{x}$ and $q_{y}$. With that in mind and in order to reduce the numerical work, we replace the $\delta$ function (25) by a Lorentzian of width $\Gamma$ and neglect in it and in the factor $f_{n k_{y} \xi}(1$ $\left.-f_{n^{\prime}, k_{y}+K_{y}+q_{y}, \xi-c_{y}\left(K_{x}+q_{x}\right)}\right)$ the terms $\propto q_{x}$ or $\propto q_{y}$. Alternatively, we may expand the $\delta$ function in powers of $q_{x}$ and $q_{y}$; then by far the leading contribution comes from the zero-order term given by Eq. (25) with $q_{x}=q_{y}=0$. In addition, we neglect the term $q^{2}$ in $U_{\mathbf{q}}$. Further, from the sum over $n^{\prime}$ we consider only the terms $n^{\prime}=n$ and $n^{\prime}=n \pm 1$; the term $n^{\prime}=n$ gives the dominant contribution, about $90 \%$. Then the integral over $u$ can be evaluated and Eq. (24) takes the form

$$
\begin{aligned}
\sigma_{y y}^{c o l} \approx & \frac{e^{2}}{h} \frac{\beta N_{I} U_{0}^{2} \Gamma}{2 \pi^{2} a_{x}} \sum_{n}\left\{(2 n+1) \int_{0}^{2 \pi} d \xi \int_{0}^{a_{x} / \ell^{2}} d k_{y}\right. \\
& \left.\times\left[D_{n, n}+(n+1) D_{n, n+1}+n D_{n, n-1}\right]\right\},
\end{aligned}
$$

where

$$
\begin{aligned}
D_{n, n^{\prime}}= & f_{n k_{y} \xi}\left(1-f_{n^{\prime}, k_{y}+K_{y}, \xi-c_{y} K_{x}}\right) /\left[\left(E_{n k_{y} \xi} \xi\right.\right. \\
& \left.\left.-E_{n^{\prime}, k_{y}+K_{y}, \xi-c_{y} K_{x}}\right)^{2}+\Gamma^{2}\right] .
\end{aligned}
$$

As a result, when $2 \pi \ell^{2} / a_{x} a_{y}=\Phi_{0} / \Phi$ is an integer, the second and third terms in the argument of the $\delta$ function in Eq. (25) vanish and entail $n=n^{\prime}$, i.e., the response is strongest when one flux quantum passes through an integral number of cells as observed. ${ }^{8,11}$ In this case the factor $\left[(\cdots)^{2}+\Gamma^{2}\right]$ in Eq. (27) becomes $\Gamma^{2}$.

A qualitative understanding of the enhancement of the collisional conductivity for integer $\alpha=\Phi_{0} / \Phi$ is as follows. In this case only scattering between the states $\left|k_{y}, \xi\right\rangle$ and $\left|k_{y}+K_{y}+q_{y}, \xi+c y\left(K_{x}, q_{x}\right)\right\rangle$ is allowed, cf. Eq. (24). These states correspond to cyclotron orbits separated by a distance $\alpha a_{x}$ which is a multiple of the lattice period. One example is shown in Fig. 5 for two orbits that encircle a unit cell. As 


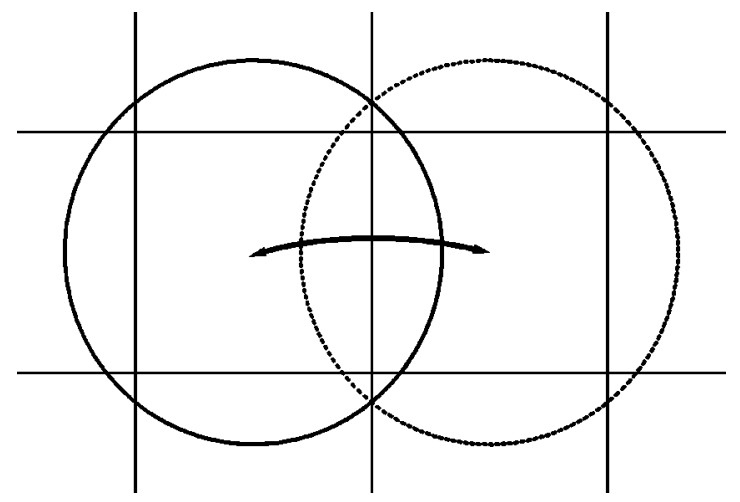

FIG. 5. Scattering between two cyclotron orbits which encircle one unit cell.

shown, the orbits are in the same relative position with respect to the modulation lattice and correspond to electron states of the same energy. Since impurity scattering is an elastic process that leads to hopping between states of the same energy, the hopping between such cyclotron orbits for integer $\alpha$ contributes the most to the conduction and enhances the collisional conductivity. On the other hand, for $\alpha$ not an integer the position of the two orbits involved in the scattering process relative to the modulation lattice changes; accordingly the enhancement mentioned above is weakened.

For those values of the magnetic field for which $F_{n}\left(u_{x}\right)$ vanishes we use the same wave functions and the spectrum (4). If the modulation periods are the same, we have $F_{n}\left(u_{x}\right)=F_{n}\left(u_{y}\right)=0, n \rightarrow n^{\prime}$, and Eq. (26) holds with $D_{n, n \pm 1} \rightarrow 0$. If the modulation periods are not equal or if $\Phi_{0} / \Phi$ is not an integer, Eqs. (25) and (26) hold only approximately. With all that in mind, the assumption that the small gaps are closed due to disorder, and for computational convenience, we use Eq. (26) as an approximation for all fields.

For the hexagonal modulation we obtain again Eq. (26) but now the energy spectrum is given by Eq. (10). Further, $a_{x}$ and $u_{y}$ are replaced by $a$ and $u=8 n^{2} \ell^{2} / 3 a^{2}$, respectively. For $\sigma_{x x}^{c o l}$ the result is given by Eq. (26) with $a_{x}$ replaced by $a$; $u$ remains the same.

The Hall conductivity. The evaluation of Eq. (17) for $\omega$ $=0$ is readily performed with the states $\left|n k_{y} \xi\right\rangle$ and the energy levels given by Eq. (3) or Eq. (10). The only difference with the previous ${ }^{3}$ calculation is that a factor $\exp \left[i\left(k_{y}\right.\right.$ $\left.\left.-k_{y}^{\prime}\right) / K_{y}\right] \delta_{\xi, \xi^{\prime}}$ appears on the right-hand side of Eq. (17) of Ref. 3 now written as $\left\langle n k_{y} \xi\left|V_{\mu}\right| n^{\prime} k_{y}^{\prime} \xi^{\prime}\right\rangle$. For rectangular symmetry we obtain $\left[\sigma_{y x}(0) \equiv \sigma_{y x}\right]$

$$
\begin{aligned}
\sigma_{y x}= & \frac{e^{2}}{h} \frac{2 \ell^{2}}{\pi a_{x}} \sum_{n}(2 n+1) \int_{0}^{a_{x} / 2 \ell^{2}} d k_{y} \\
& \times \int_{0}^{2 \pi} d \xi \frac{f_{n k_{y} \xi}-f_{n+1, k_{y} \xi}}{\left[1+\lambda_{n x} \cos \left(K_{x} x_{0}\right)+\lambda_{n y} \cos \xi\right]^{2}},
\end{aligned}
$$

where $\lambda_{n \mu}=V_{\mu} e^{-u_{\mu} / 2} L_{n+1}^{-1}\left(u_{\mu}\right) / \hbar \omega_{c}, \mu=x, y$. We notice that for $V_{y}=0$ we obtain the previous $1 \mathrm{D}$ result. ${ }^{3}$ We also remark that Eq. (28) is valid for hexagonal symmetry with

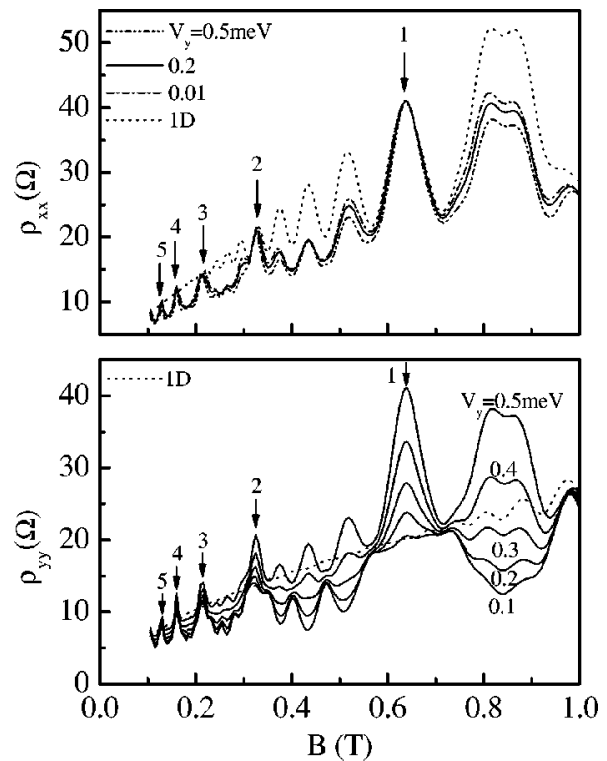

FIG. 6. Resistivity components $\rho_{x x}$ and $\rho_{y y}$ as a function of the magnetic field $B$ for fixed $V_{x}=0.5 \mathrm{meV}$ and varying $V_{y}$. The dotted curve is the 1D limit obtained with $V_{y}=0$. The prominent peaks in the 2D case are marked by the integral value of $\alpha=\Phi_{0} / \Phi$.

$a_{x} \rightarrow a, u_{x}=u_{y}=u=8 \pi^{2} \ell^{2} / 3 a^{2}$, and of course the different energy levels [Eq. (10)] that enter the factor $f_{n k_{y} \xi}$.

\section{NUMERICAL RESULTS}

We now present results for the various resistivity components $\rho_{\mu \nu}$ using the standard expressions given at the end of Sec. III A, and evaluating numerically the conductivities given by Eqs. (18), (26), and (27). For Figs. 3-7, and 10 we use the parameters of Ref. 11. They are electron density $n_{s}$ $=4.5 \times 10^{15} / \mathrm{m}^{2}$, temperature $T=5.5 \mathrm{~K}$ or $T=1.6 \mathrm{~K}, a_{x}$ $=a_{y}=804 \AA$, and mobility $\mu=70 \mathrm{~m}^{2} / \mathrm{V} \mathrm{s}$. The corresponding parameters for Figs. 8 and 9, taken from Ref. 5, are $n_{s}$
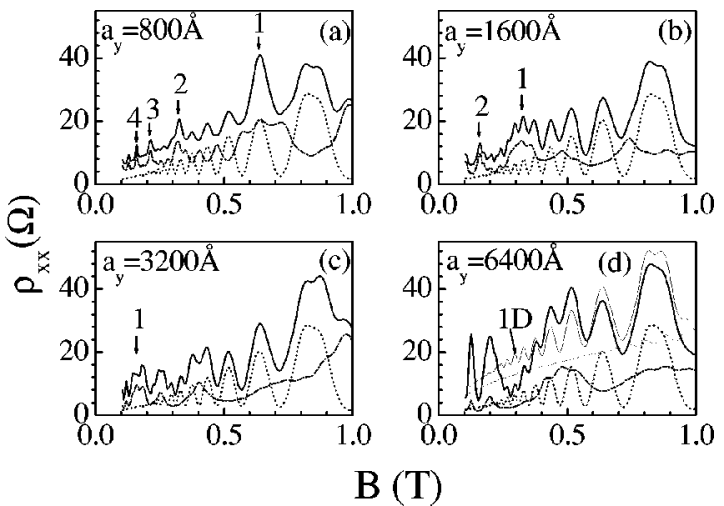

FIG. 7. Resistivity component $\rho_{x x}$ as a function of the magnetic field $B$ for fixed $V_{x}=V_{y}=0.5 \mathrm{meV}$ and different periods $a_{y}$ as indicated. The solid curves give the total resistivity, the dotted ones the diffusive contribution, and the dash-dotted ones the collisional contribution. The thinner curves in panel (d) are for the 1D limit $\left(V_{y}=0\right)$. The prominent peaks in the $2 \mathrm{D}$ case are marked by the integral values of $\alpha$. 


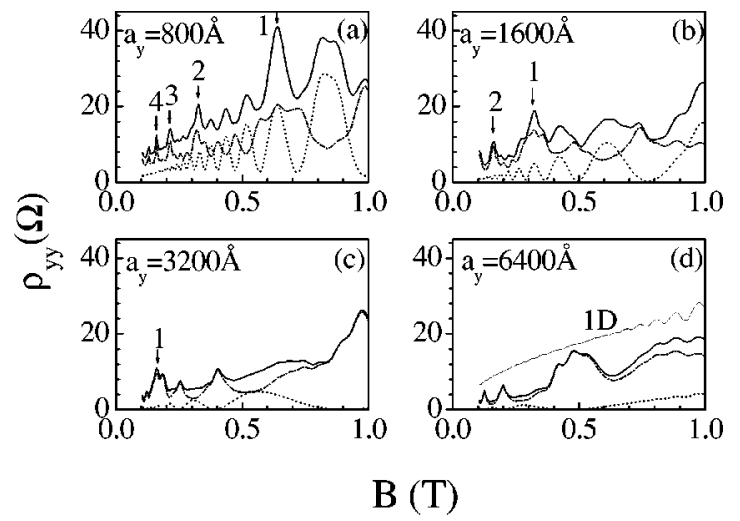

FIG. 8. The same as in Fig. 7 but for the resistivity component $\rho_{y y}$.

$=5.1 \times 10^{15} / \mathrm{m}^{2}$, temperature $T=4.2 \mathrm{~K}, a_{x}=a_{y}=2820 \AA$, and mobility $\mu=140 \mathrm{~m}^{2} / \mathrm{Vs}$. The relaxation time at zero magnetic field, $\tau_{0}$, is estimated from the sample mobility as $\tau_{0}=m^{*} \mu / e$. Then the level width is $\Gamma=\left(e B N_{I} U_{0}^{2} / \pi \hbar\right)^{1 / 2}$ $=\left(e \hbar / m^{*}\right) \sqrt{B / \pi \mu}$.

In Fig. 6 we plot $\rho_{x x}$ and $\rho_{y y}$ as a function of the magnetic field $B$ with $V_{x}=0.5 \mathrm{meV}$ for constant $\tau=\tau_{0}$. As indicated, the various curves correspond to different $V_{y}$ and the dotted one, marked 1D, represents the 1D limit obtained with $V_{y}$ $=0$. The prominent peaks in the $2 \mathrm{D}$ case, marked by the integral value of $\alpha$, result from the collisional contribution to the conductivity. The smaller peaks, between these values of $\alpha$, correspond to the commensurability or Weiss oscillations. Notice how the prominent peaks of $\rho_{x x}$, in the 2D case, remain rather insensitive to changes in $V_{y}$ : this is so because they result from the collisional contribution $\sigma_{y y}^{c o l}$ which depends very weakly on $V_{y}$ through the energy spectrum. The apparently drastic difference between the two figures results from the fact that $\sigma_{\mu \mu} \ll \sigma_{x y}$ makes $S$ change little and $\rho_{x x}$ $=\sigma_{y y} / S$ while $\rho_{y y}=\sigma_{x x} / S$. Upon reducing $V_{y}$ the contribution $\sigma_{x x}^{d i f} \sim V_{y}^{2}$, given by Eq. (18), is affected drastically whereas $\sigma_{y y}^{c o l}$ is not.

In Figs. 7 and 8 we plot again $\rho_{x x}$ and $\rho_{y y}$ as a function of the field $B$ with $V_{x}=V_{y}=0.5 \mathrm{meV}$, for the same $\tau$ and $\Gamma$

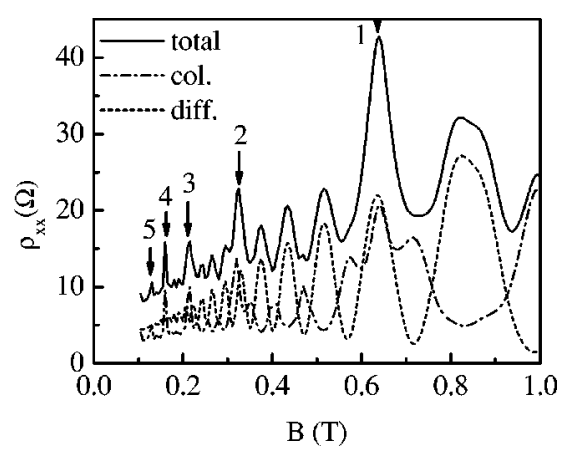

FIG. 9. Resistivity component $\rho_{x x}$ as a function of the magnetic field $B$ for fixed $V_{x}=V_{y}=1 \mathrm{meV}$ and $T=5.5 \mathrm{~K}$. The solid curves give the total resistivity, the dotted ones the diffusive contribution, and the dash-dotted ones the collisional contribution. The prominent peaks are marked by the integral values of $\alpha=\Phi_{0} / \Phi$.

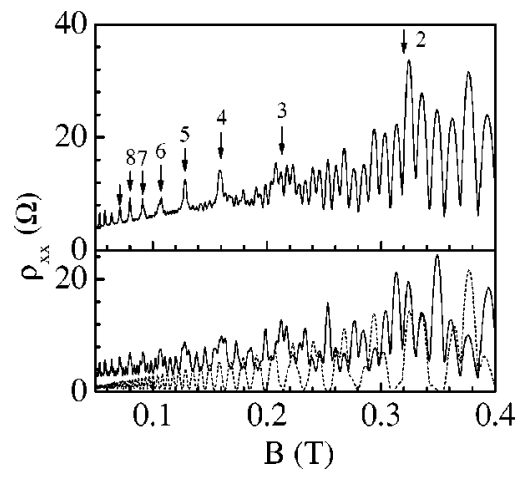

FIG. 10. The same as in Fig. 9 but for temperature $T=1.6$. The lower panel shows the collisional contribution (solid curve) and the diffusive one (dotted curve); the upper panel shows their sum.

$\propto B^{1 / 2}$ as in Fig. 6 but with the period $a_{y}$ being doubled from panel to panel as indicated. The solid curves give the total resistivity, the dashed ones the diffusive contribution, defined by $\rho_{\mu \mu}^{d i f}=\sigma_{\nu \nu}^{d i f} / S$, and the dotted ones the collisional contribution, defined by $\rho_{\mu \mu}^{c o l}=\sigma_{\nu \nu}^{c o l} / S$. Notice how the prominent peaks move to lower fields with increasing $a_{y}$ as explained after Eq. (26); in panel (d) they have disappeared. The 1D limit shown in panel (d) is obtained with $V_{y}=0$ and the difference in the $B$ dependence between $\rho_{x x}$ and $\rho_{y y}$ is related to that of the corresponding conductivity contributions. One of them, $\sigma_{x x}^{d i f}$ given Eq. (18), is affected drastically by changing $V_{y}$ and/or the period $a_{y}$ which enters the factor $\sin ^{2} \xi$, the others very weakly.

We now look more closely at the experimental results of Refs. 8 and 5 with the parameter sets specified above. The parameters $V_{x}$ and $V_{y}$ are not known. In Fig. 6 we have shown the total resistivity for $V_{x}=V_{y}=0.5 \mathrm{meV}$, constant $\tau$ $=\tau_{0}$. In Fig. 9 we plot again $\rho_{x x}$ versus $B$ but now we show the contributions $\rho_{x x}^{\text {dif }}$ and $\rho_{x x}^{c o l}$ as well. In addition, we take $V_{x}=V_{y}=1 \mathrm{meV}$, and $1 / \tau \sim \Gamma \sim B^{1 / 2}$. Because $\sigma_{\nu \nu}^{d i f} \ll \sigma_{\nu \nu}^{c o l}$, the difference in the total resistivity is very small between the two sets of modulation strengths. However, the oscillation amplitudes in $\rho_{\mu \mu}^{c o l}$ are higher in the present case and $\rho_{x x}$ increases more slowly with $B$ as observed. ${ }^{8}$ Upon closer inspection we see that the prominent peaks, marked by the integral values of $\alpha$, result entirely from the collisional contribution $\sigma_{\nu \nu}^{c o l}$. As can be seen in Fig. 3 of the following paper, ${ }^{11}$ the amplitudes and positions of these peaks agree well with the experimental results. Notice also how the Weiss oscillations of $\rho_{x x}^{d i f}$ and $\rho_{x x}^{c o l}$, between these peaks, are in antiphase. These experimental results for $\rho_{y y}$ and $\rho_{x x}$ are for two orthogonal crystal directions [011] and [01 $\overline{1}]$ taken from different samples. They have similar structures and the curves may be fitted theoretically using slightly different potentials. A direct comparison between experimental and theoretical results is made in Fig. 9 of the following paper ${ }^{11}$ for $\tau=\tau_{0}$ but qualitatively the agreement is the same for $\tau$ $\propto B^{-1 / 2}$. Notice, however, that the theoretical oscillation amplitudes and the overall value of $\rho_{x x}$ in the low- $B$ region, below $\alpha=2$, agree less well with the experimental ones than those in the high- $B$ region.

In Ref. 11 results are given for temperature $1.6 \mathrm{~K}$ and 


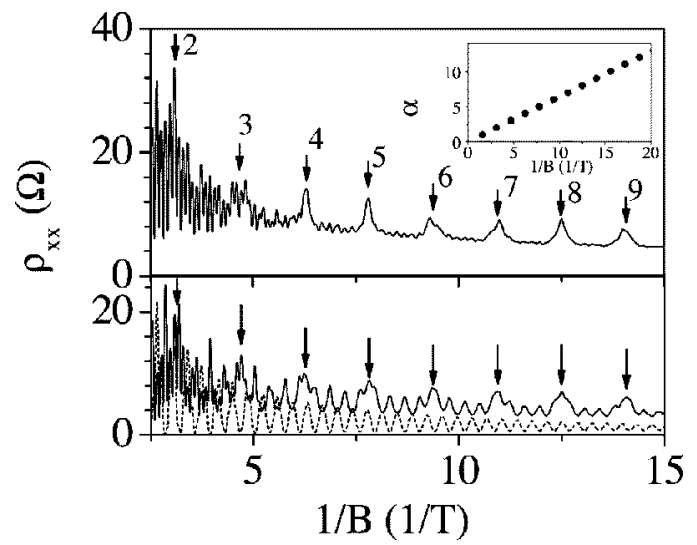

FIG. 11. Resistivity component $\rho_{x x}$ as a function of inverse magnetic field $1 / B$. The curves are marked as in Fig. 10. The inset shows the peak position versus $1 / B$.

otherwise the same parameters. We show the calculated $\rho_{x x}$ for this case in Fig. 10. As can be seen, lowering the temperature makes visible all prominent peaks marked by arrows for $\alpha=1, \ldots, 8$. Their positions occur at fields $B$ $=0.64,0.32,0.21,0.16,0.13,0.11,0.09$, and $0.08 \mathrm{~T}$ and compare very well with the experimental ones, see Fig. 6 in the following paper. ${ }^{11}$ To see more clearly the oscillations we replot, in Fig. 11, the resistivities in the low-field region of Fig. 10 as a function of $1 / B$.

The temperature dependence of the oscillations is shown in Fig. 12. The solid, dotted, and thin solid curves correspond to $T=5,10$, and $20 \mathrm{~K}$, respectively. As can be seen, these new oscillations are more robust than the Weiss oscillations and persist at $T=20 \mathrm{~K}$. However, their damping with $T$ is weaker than the observed one. ${ }^{11}$

In Fig. 13 we plot $\rho_{x x}$ in the manner of Fig. 9 but for the parameters of Ref. 5 involving the much longer periods $a_{x}$ $=a_{y}=2820 \AA$. The modulation strengths are $V_{x}=V_{y}$ $=0.2 \mathrm{meV}$ and very close to those used in Refs. 5 and 6 . The

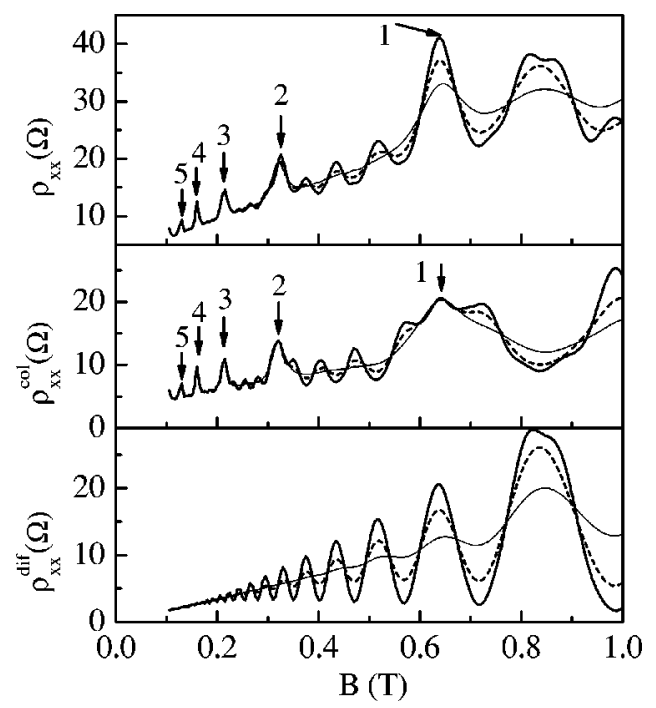

FIG. 12. Resistivity component $\rho_{x x}$ as a function of the magnetic field $B$. The solid, dotted, and thin solid curves correspond to $T=5,10$, and $20 \mathrm{~K}$, respectively.

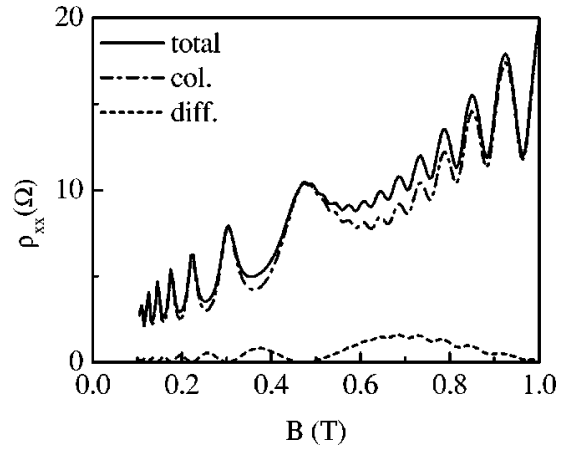

FIG. 13. The same as in Fig. 9 with the parameters of Ref. 5 and $V_{x}=V_{y}=0.2 \mathrm{meV}$.

agreement with the experimental 2D results of Ref. 5 is very good: below approximately $B=0.5 \mathrm{~T}$ we have the Weiss oscillations and above it the Shubnikov-de Haas ones. One noticeable feature here is the absence of the prominent peaks for integral values of $\alpha$. This is so because the much longer periods involved make $\alpha=2 \pi \ell^{2} / a_{x} a_{y}$ integer for much smaller values of $B$. For instance, $\alpha=1$ occurs at $B$ $=0.05 \mathrm{~T}$ and the corresponding peak is not resolved. The agreement is also as good if we use $a_{x}=a_{y}=3650 \AA$ and otherwise the same parameters pertaining to another sample.

Reference 5 reported results also for 1D modulations. As mentioned earlier, we can obtain the 1D limit from the present $2 \mathrm{D}$ results by considering a vanishing $V_{y}$. In Fig. 14 we show the 1D limit of the total $\rho_{x x}$ and $\rho_{y y}$ for $a_{x}$ $=2820 \AA, V_{x}=0.5 \mathrm{meV}$, and $V_{y}=0$. Although the agreement between theory and experiment is very good, it must be noticed that it is obtained with $V_{x}=0.5 \mathrm{meV}$ and not $V_{x}$ $=0.2 \mathrm{meV}$ that we used in Fig. 13. Since the $1 \mathrm{D}$ or $2 \mathrm{D}$ modulations are produced by illumination of the samples, we expect them to have the same strength. If we use $V_{x}$ $=0.2 \mathrm{meV}$ we can obtain good agreement if we use a $\tau$ smaller by about a factor of 2 in Eq. (18). As stated in Refs. 5 and 6 , this may be an indication that in this very high mobility samples the fine structure of the energy spectrum, which the present theory neglects, is partially resolved. However, the experimental data were taken at $T=4.2 \mathrm{~K}$ and, as no such fine structure has been observed above millikelvin temperatures, alternative explanations have been proposed. ${ }^{4,11}$

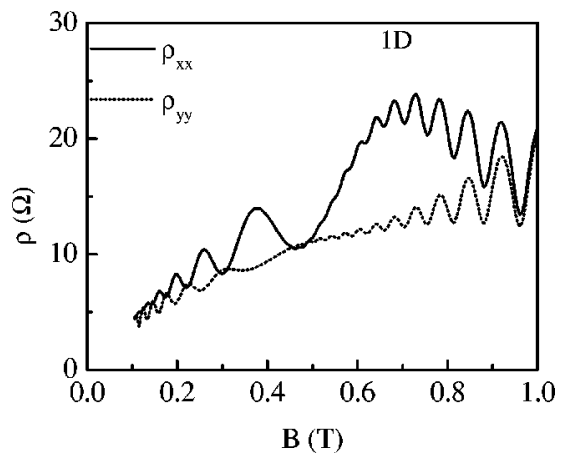

FIG. 14. The 1D resistivity component $\rho_{\mu \mu}$ as a function of the magnetic field $B$ obtained with $a_{x}=2820 \AA, V_{x}=0.5 \mathrm{meV}$, and $V_{y}=0$. 


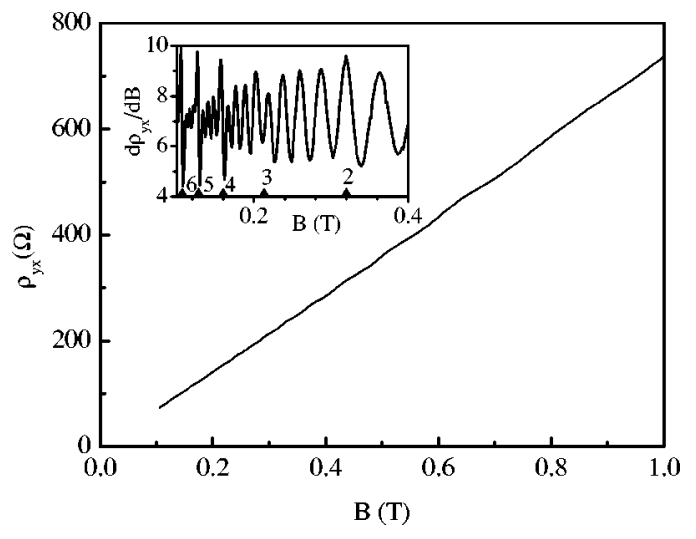

FIG. 15. The Hall resistivity $\rho_{y x}$ vs magnetic field $B$ with the parameters of Fig. 9. The inset shows the derivative $d \rho_{y x} / d B$ vs $B$.

Finally, in Fig. 15 we show the Hall resistivity $\rho_{y x}$ for the parameters of Fig. 9. As in the case of 1D modulations, it exhibits very weak oscillations. They are better seen in the inset which shows the derivative $d \rho_{y x} / d B$ versus $B$. The triangles on the $x$ axis mark the positions of the integral values of $\alpha$ for which enhanced oscillations are observed.

\section{CONCLUDING REMARKS}

We presented a theory of magnetotransport in 2D superlattices using the energy spectrum and wave functions that result from the tight-binding difference equation when the parameter $\alpha=\Phi_{0} / \Phi$ is an integer. As emphasized in the text and supported with the results for the DOS shown in Fig. 4, the description holds approximately for all fields if we assume that the small gaps in the energy spectrum are closed due to disorder. The reasonable-to-good agreement with the experimental results strongly supports this assumption.

As detailed in the text, the prominent peaks, for $\alpha$ $=\Phi_{0} / \Phi$ integer, result from the collisional contribution to the conductivity $\sigma_{y y}^{c o l}$, require sufficiently short periods, and depend very weakly on the value of the modulation strengths $V_{x}$ and $V_{y}$. Upon increasing the period along one direction we showed how they move to lower fields. Accordingly, for periods between $3000 \AA$ and $4000 \AA$ these peaks occur at much smaller magnetic fields and are not resolved. ${ }^{5}$ The agreement between our results and the experimental ones, as presented in Ref. 8 and detailed in the preceding paper, ${ }^{11}$ is good for the peak positions at all fields. The oscillation amplitudes agree well at relatively high fields but less well at low fields. As shown in Fig. 12, these oscillations are quite robust with respect to the temperature but their damping with temperature is weaker than the observed one.

Between the oscillations for $\alpha=\Phi_{0} / \Phi$ integer we have the Weiss oscillations. The relative phase between those of $\rho_{x x}$ and those of $\rho_{y y}$ depends on the values of the modulation strengths, cf. Fig. 6 in which the period is the same for all curves, and of the modulation periods, cf. Figs. 4 and 5 in which the modulation strengths are the same for all panels. We notice that as $V_{y}$ becomes smaller and smaller than $V_{x}$, the oscillations resemble more closely those corresponding to $1 \mathrm{D}$ weak modulations, ${ }^{15} \mathrm{cf}$. Fig. 6 . The results for the latter can be extracted from the present $2 \mathrm{D}$ ones if we take the modulation strength along one direction to be zero.

The relative phase between $\rho_{x x}$ and $\rho_{y y}$ for 1D and 2D modulations depends strongly on the ratio of the modulation strengths $V_{x}$ and $V_{y}$. Since one resistivity component vanishes for 1D modulations, this conclusion could not be reached by studying only the latter. Similar results were reported in Ref. 6.

\section{ACKNOWLEDGMENTS}

This work was supported by the Canadian NSERC Grant No. OGP0121756, the Belgian Interuniversity Attraction Poles (IUAP), the Flemish Concerted Action (GOA) Program, and the EU-CERION program. We also thank A. Long and J. H. Davies for stimulating discussions and important clarifications concerning the experimental results of the following paper.
${ }^{1}$ D. Weiss, K. von Klitzing, K. Plog, and G. Weimann, Europhys. Lett. 8, 179 (1989); R.W. Winkler, J.P. Kotthaus, and K. Ploog, Phys. Rev. Lett. 62, 1177 (1989).

${ }^{2}$ R.R. Gerhardts, D. Weiss, and K. von Klitzing, Phys. Rev. Lett. 62, 1173 (1989); C.W.J. Beenakker, ibid. 62, 2020 (1989); P. Vasilopoulos and F.M. Peeters, ibid. 63, 2120 (1989); H.L. Cui, V. Fessatidis, and N. Horing, ibid. 63, 2598 (1989); P. Streda and A.H. MacDonald, Phys. Rev. B 41, 11892 (1990); C. Zhang and R.R. Gerhardts, ibid. 41, 12850 (1990); F.M. Peeters and P. Vasilopoulos, ibid. 42, 5899 (1990).

${ }^{3}$ F.M. Peeters and P. Vasilopoulos, Phys. Rev. B 46, 4667 (1992).

${ }^{4}$ (a) D.E. Grant, A.R. Long, and J.H. Davies, Phys. Rev. B 61, 13 127 (2000); (b) F. M. Peeters and P. Vasilopoulos, Proceedings of the 20th International Conference on the Physics of Semiconductors, edited by E. M. Anastassakis and J. D. Joannopoulos (World Scientific, Singapore, 1990), Vol. 2, p. 1589.

${ }^{5}$ R.R. Gerhardts, D. Weiss, and U. Wulf, Phys. Rev. B 43, 5192 (1991).
${ }^{6}$ D. Pfannkuche and R.R. Gerhardts, Phys. Rev. B 46, 12606 (1992).

${ }^{7}$ E.S. Alves, P.H. Beton, M. Henini, L. Eaves, P.C. Main, O.H. Hughes, G.A. Toombs, S.P. Beaumont, and C.D.W. Wilkinson, J. Phys.: Condens. Matter 1, 8257 (1989); H. Fang and P.J. Stiles, Phys. Rev. B 41, 10171 (1990); A. Toriumi, K. Ismail, M. Burkhardt, D.A. Antoniadis, and Henry I. Smith, ibid. 41, 12346 (1990).

${ }^{8}$ S. Chowdhury, A. R. Long, J. H. Davies, K. Lister, and E. Skuras, Proceedings of EP2DS-14, p. 583 (2001).

${ }^{9}$ S. Chowdhury, C.J. Emeleus, B. Milton, E. Skuras, A.R. Long, J.H. Davies, G. Pennelli, and C.R. Stanley, Phys. Rev. B 62, R4821 (2000); P. Rotter, M. Suhrke, and U. Rössler, ibid. 54, 4452 (1996); C. Albrecht, J.H. Smet, D. Weiss, K. von Klitzing, R. Hennig, M. Suhrke, U. Rossler, V. Umansky, and H. Schweizer, Phys. Rev. Lett. 86, 147 (2001).

${ }^{10}$ J. Labbé, Phys. Rev. B 35, 1373 (1987). 
${ }^{11}$ S. Chowdhury, A. R. Long, E. Skuras, J.H. Davies, K.Lister, G. Pennelli, and C.R. Stanley, preceding paper, Phys. Rev. B 69, 035330 (2004).

12 (a) D.R. Hofstadter, Phys. Rev. B 14, 2239 (1976); (b) F.H. Claro and G.H. Wannier, ibid. 19, 6068 (1979).

${ }^{13}$ N.A. Usov, Zh. Éksp. Teor. Fiz. 94, 305 (1988) [Sov. Phys. JETP 67, 2565 (1988)].

14 (a) P. Vasilopoulos and C.M. Van Vliet, J. Math. Phys. 25, 1391 (1984); (b) Phys. Rev. B 34, 4375 (1986); (c) P. Vasilopoulos, ibid. 32, 771 (1985); (d) 34, 3019 (1986).

${ }^{15}$ For recent studies of strong $1 \mathrm{D}$ modulations and the relevant observations or predictions, we refer the reader to the following works: J. Shi, F.M. Peeters, K.W. Edmonds, and B.L. Gallagher, Phys. Rev. B 66, 035328 (2002); M. Langenbuch, M. Suhrke, and U. Rössler, Europhys. Lett. 61, 520 (2003); K. Vyborny, L. Smrcka, and R.A. Deutschmann, Phys. Rev. B 66, 205318 (2002). 